\title{
ALTERNATIVA ESTÉTICA Y FUNCIONAL DEL SECTOR ANTERIOR EN DENTICIÓN DECIDUA
}

ESTHETIC AND FUNCTIONAL ALTERNATIVE OF THE ANTERIOR SECTOR IN DECIDUOUS DENTITION

\section{RESUMEN}

Alarcón-Calle, Catherine Sharlot ${ }^{1}$

Perona-Miguel de Priego, Guido

Mock-Ferreyros, Augusto $^{3}$

El tratamiento odontológico del niño con dentición decidua tiene como fin la preservación e integridad de la cavidad oral. Son muchas las causas que desencadenan la ausencia dentaria. La pérdida prematura de manera individual o múltiple puede generar trastornos como: limitación en funciones básicas, disminución de la longitud de los arcos dentarios, aparición de hábitos incorrectos y llegar al punto de disminuir la autoestima del niño. La prótesis fija con bandas ortodóncicas es una opción para obtener un recurso funcional y estético en niños que son poco colaboradores para usa aparatos removibles.

El presente caso tuvo como objetivo preservar el tejido de sostén y mucosa oral; para que de esa manera las funciones de masticación, deglución y fonación no se vean alteradas; proveer una estética armónica dentofacial lo cual evitará la extrusión de los dientes antagonistas al espacio edéntulo y recuperar la autoestima perdida.

Palabras clave: prótesis fija, dentición decidua

\section{ABSTRACT}

The dental treatment of the child with deciduous dentition is aimed at the preservation and integrity of the oral cavity. There are many causes that tooth absence. The premature loss of individual or multiple ways can generate disorders such as limitation in basic functions, decrease in the length of the dental arches, appearance of incorrect habits and reach the point of diminishing the child's self-esteem. The fixed prosthesis with orthodontic bands is an option to obtain a functional and aesthetic appeal in children who are not very cooperative to use removable appliances.

The objective of this case was: to preserve the supporting tissue and oral mucosa; so that in this way the functions of chewing, swallowing and phonation are not altered; provide a dentofacial harmonic aesthetic which will prevent the extrusion of opposing teeth into the edentulous space and recover lost self-esteem.

Keywords: fixed prosthesis, deciduous dentition

\footnotetext{
Citar como:

Alarcón Calle CS, Perona-Miguel de Priego G, Mock-Ferreyros A. Alternativa estética y funcional del sector anterior en dentición decidua. Rev Cient Odontol 2017; 5 (2): 772-9.

${ }^{1}$ Egresada Especialidad Odontopediatría UCSUR.

${ }^{2}$ Mg. en Odontopediatría UCSUR. Especialista en Odontología Pediátrica UPCH. Coordinador de la Maestría en Odontopediatría UCSUR.

${ }^{3}$ Mg. Especialista en Odontopediatría UAG. Docente Especialidad Odontopediatría UCSUR.
} 


\section{INTRODUCCIÓN}

La presencia de caries aparición temprana severa, caries de aparición temprana, traumatismos dentales, enfermedad periodontal, defectos de la estructura dentaria; son algunas de las causas por las cuales un niño presenta pérdida o ausencia de piezas dentarias(1-3).

La Asociación Dental Americana (ADA), el Centro de Prevención y Control de Enfermedades de los EE. UU. (CDC) y la Asociación de Odontopediatría (AAPD) definen a la caries de infancia temprana o early childhood caries - ECC (en inglés) como cualquier tipo de lesión de caries presente en niños menores de 6 años y a la caries severa en la infancia o S-ECC cuando la lesión de caries se inicia en los incisivos deciduos superiores(4-6). La falta de atención oportuna en niños ocasiona una serie de secuelas como son infecciones, problemas estéticos, dificultades para la alimentación, alteraciones del lenguaje y aparición de maloclusiones y hábitos orales, que conllevan a la perdida prematura de las piezas dentarias(1-4,6,7).

Durante la etapa de dentición decidua son frecuentes las caídas, fracturas dentarias, avulsión o desplazamiento dentario, daño en el tejido periodontal que requiere en la mayoría de casos la extracción dentro del plan de tratamiento $(8,9)$.

Enfermedad periodontal a temprana edad presenta un incidencia menor que la caries dental y traumatismos dentarios; pero cuando esta se manifiesta suele ser muy agresiva produciendo la exfoliación prematura de dientes deciduos en donde la eliminación del agente causal y un control no logró ser oportuno dando paso a la rehabilitación protésica. ${ }^{1}$ A su vez los defectos congénitos desarrollados en esmalte o dentina dan lugar a patologías severas y de progresión rápida como: amelogénesis imperfecta, dentinogénesis imperfecta y odontodisplasias en las cuales el tratamiento restaurador con técnicas adhesivas no es factible(10-12).

Los objetivos del tratamiento protésico son:

- Restablecer la función masticatoria: el paciente infante está en pleno crecimiento y desarrollo el cual va de la mano con la alimentación. La alteración de esta función conlleva a la alteración de su nutrición y crecimiento general(1-3).

- Permitir la correcta deglución: las alteraciones aparecen cuando la pérdida dentaria se produce en el sector anterior y el colocar una prótesis permite una correcta posición de la lengua(11).

- Proveer una estética armónica dento-facial: la ausencia de piezas dentales en el sector anterior repercute en la imagen del niño, ya que suele verse diferente a sus compañeros y puede ser motivo de burlas(12-15).

- Evitar alteraciones en la fonación: la mayoría de los sonidos se producen cuando la lengua toca la superficie lingual de los incisivos superiores. Los fonemas afectados suelen ser las consonantes: "s", " $z$ ", , "v", y "f". Los fonoaudiólogos indican que las repercusiones son mayores en niños que todavía no han adquirido el lenguaje(16,17).

- Evitar la aparición de hábitos incorrectos: cuando el niño siente un vacío en el sector anterior tiende a ocuparlo colocando la lengua en dicha zona; logrando una succión inadecuada y logrando el desplazamiento de dientes aledaños(18). 
- Mantener el espacio e evitar la extrusión del o los dientes antagonistas al espacio edéntulo(19,20).

- Preservar los tejidos de sostén y mucosa oral(1$3,19,20)$.

Las prótesis están indicadas en pérdida temprana de dientes deciduos, cuando la estética es de importancia, cuando la radiografía nos revela que las piezas permanentes tardaran algún tiempo en erupcionar y el niño por lo menos tenga una edad de dos años y medio(2). Por esta razón y con base a una revisión de la literatura fue posible establecer la importancia de

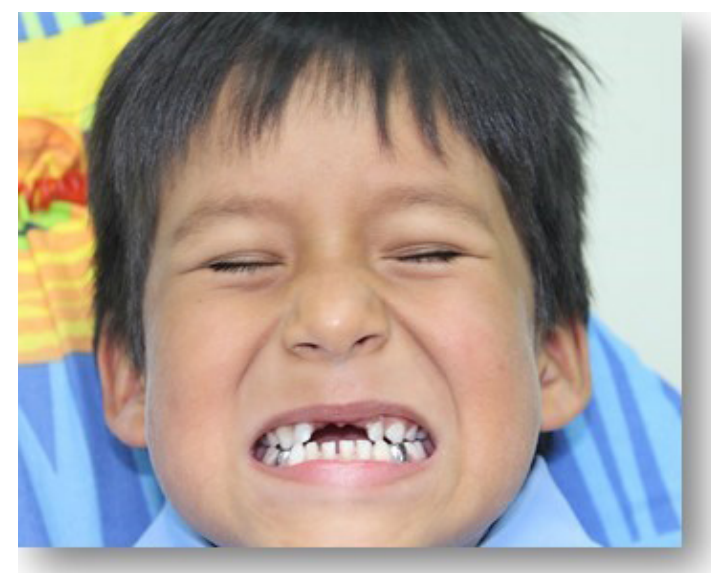

Figura 1. Vista frontal del paciente al momento del examen odontológico.

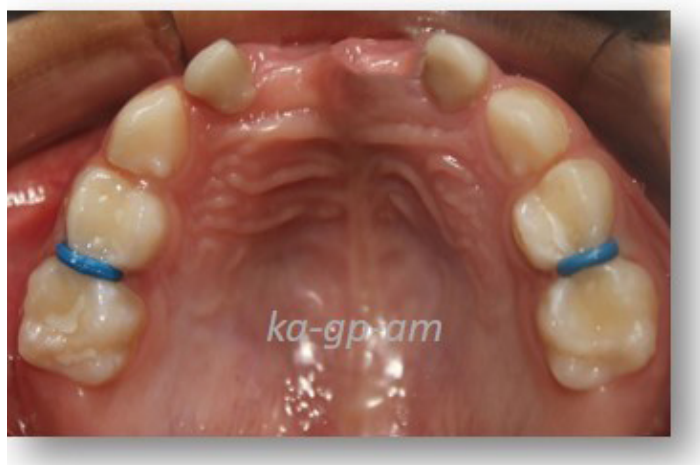

Figura 3. Cita 1. Vista oclusal luego de la colocación de ligas separadoras. la odontología protésica como una alternativa que permita el restablecimiento de sus funciones y estética adecuadamente.

\section{Relato de caso}

Paciente sexo masculino de 3 años de edad y 7 meses que acude a consulta por baja autoestima a causa de pérdida temprana de dientes anteriores. Después de una evaluación odontológica, se decide confeccionar una prótesis fija anterior soldada a bandas, esto sirvió para mejorar la autoestima del paciente.

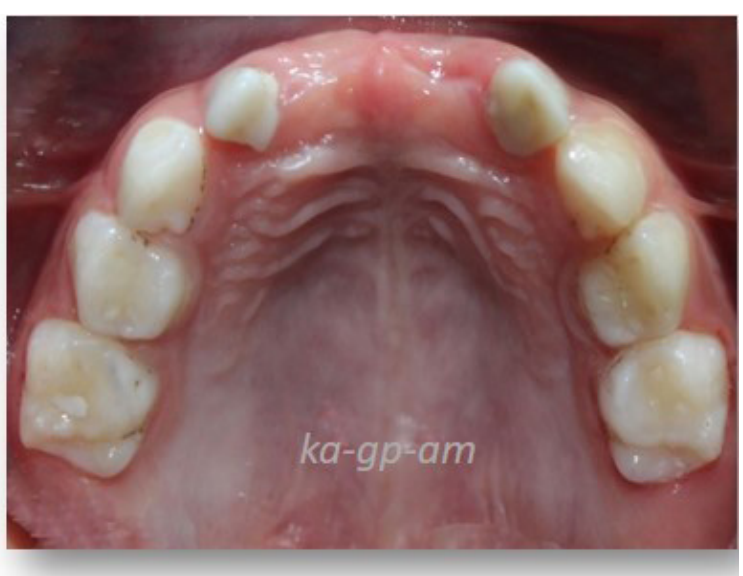

Figura 2. Vista frontal del paciente al momento del examen odontológico.

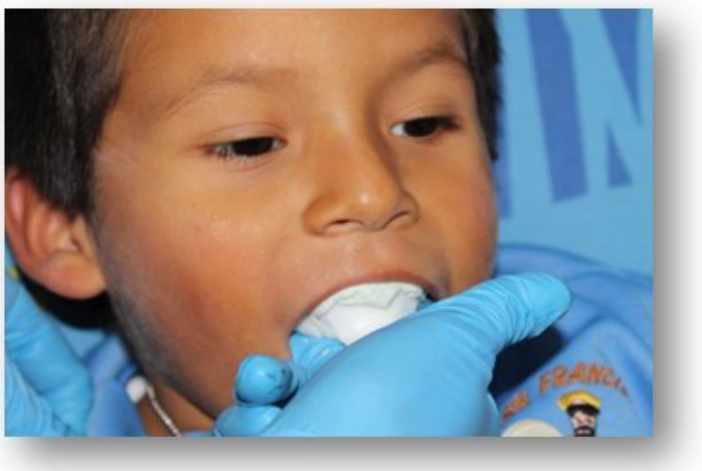

Figura 4. Cita 2. Adaptación de las bandas ortodóncicas y toma de impresión de arrastre. 


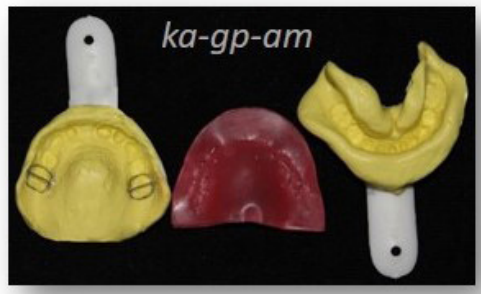

Foto 5. Cita 2. Impresión de Alginato del Arcada superior con bandas sujetadas, arcada inferior y registro de mordida.

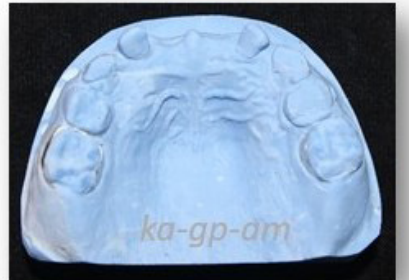

Foto 6. Cita 2. Modelo superior en yeso piedra azul con bandas.

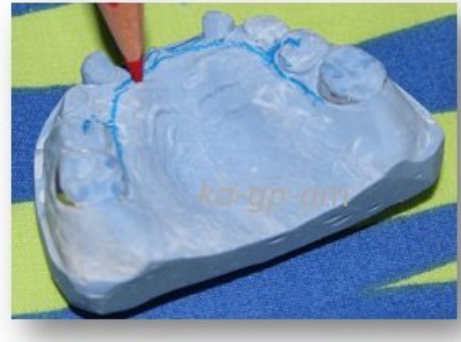

Foto 7. Se dibuja en azul la zona donde irá el alambre ortodóntico duro 0,9 mm marca: Morelli.

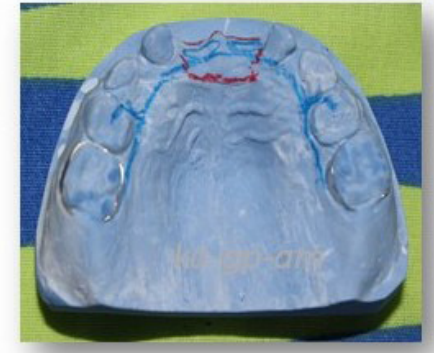

Foto 8. Se dibuja en rojo la zona donde irán los dientes artificiales y el acrílico marca Morelli.

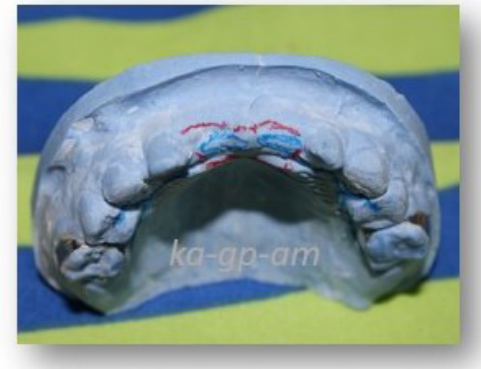

Foto 9. Se debe cuidar que la zona de acrílico no sea muy extensa por vestibular.

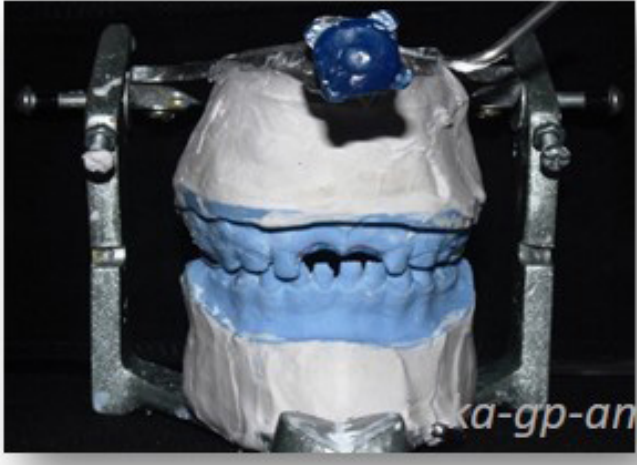

Figura 10. Para tener una referencia de su oclusión se debe montar los modelos en un oclusor.

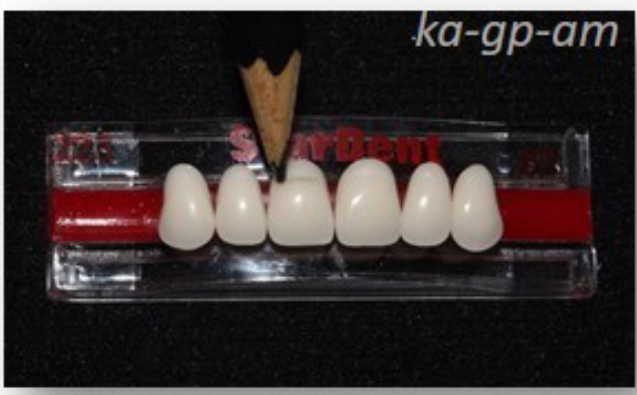

Figura 12. En este caso usamos dientes de stock de adulto marca Stardent color 59.

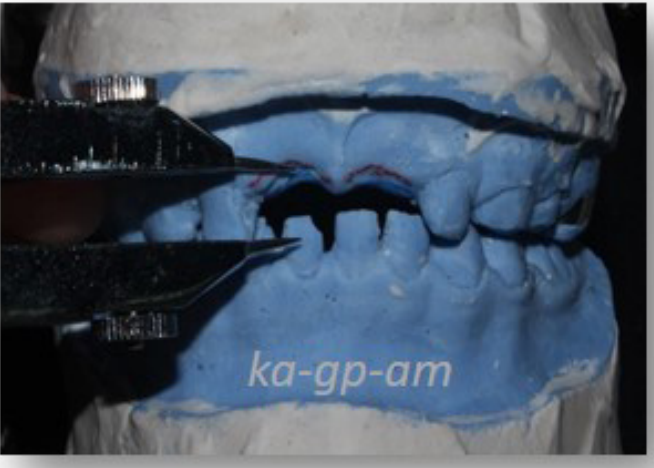

Figura 11. Con ayuda de un compás con doble punta seca se proyecta el diente a reemplazar y se anota la longitud.

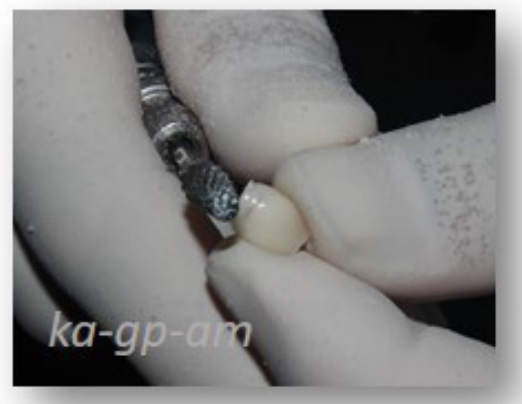

Figura 13. Una vez marcada la longitud del diente, se comienza a desgastar con fresón para degastar acrílico y micromotor. 


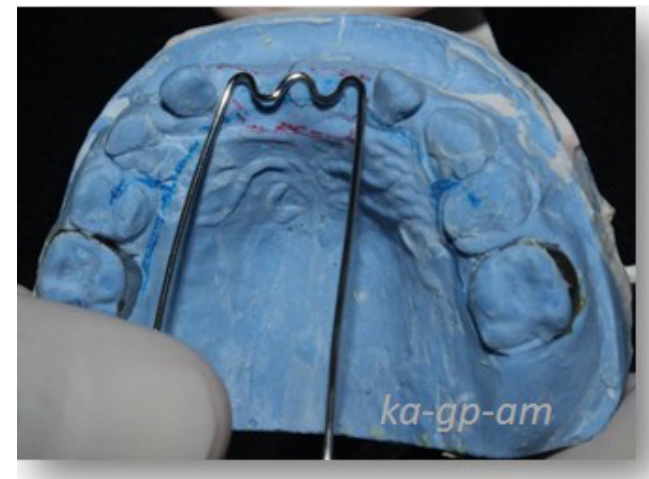

Figura 14. Con el alambre ortodóntico comenzamos a realizar las retenciones.

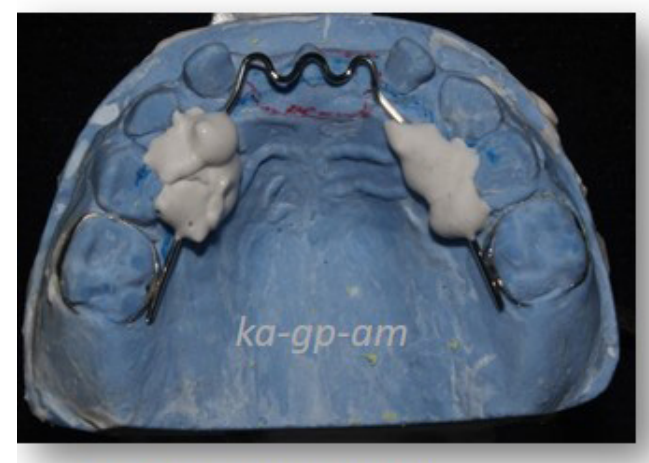

Figura 16. Se procede a fijar el alambre con yeso parís.

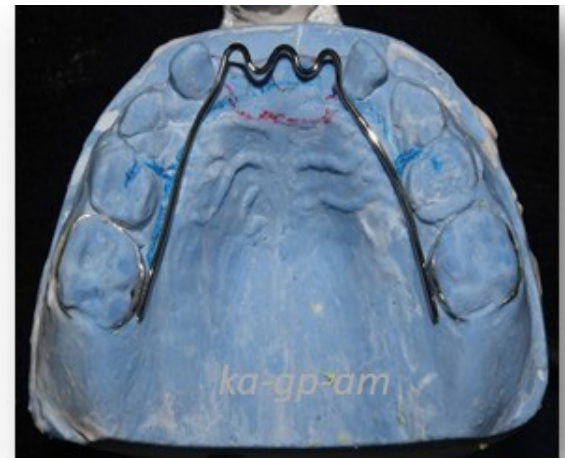

Figura 15. Luego procedemos a contornear el alambre rodeando la arcada hasta llegar a las bandas.

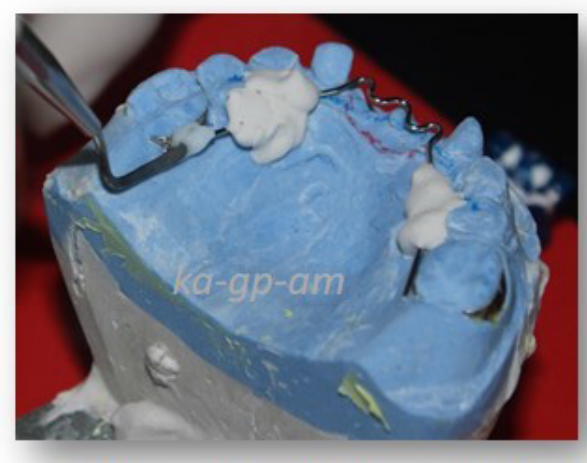

Figura 17. Se coloca el fundente marca Morelli entre la banda y el alambre en ambos lados.

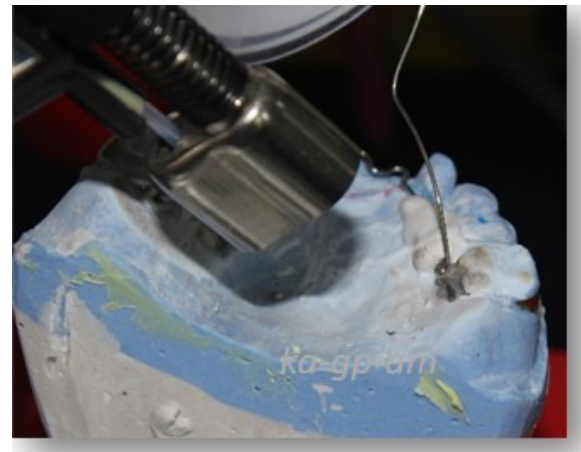

Figura 18. Se fija con alambre de soldadura marca Morelli.

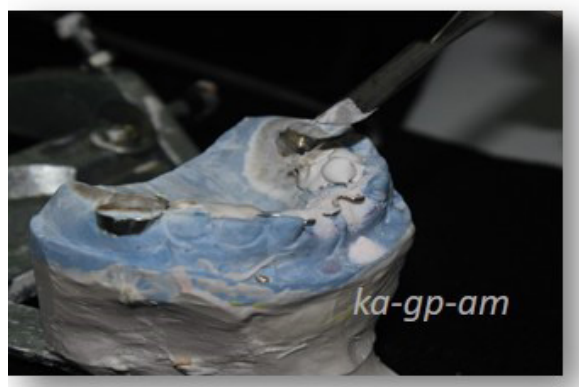

Figura 21. Colocar el acrílico en polvo de color rosado para la falsa encía.

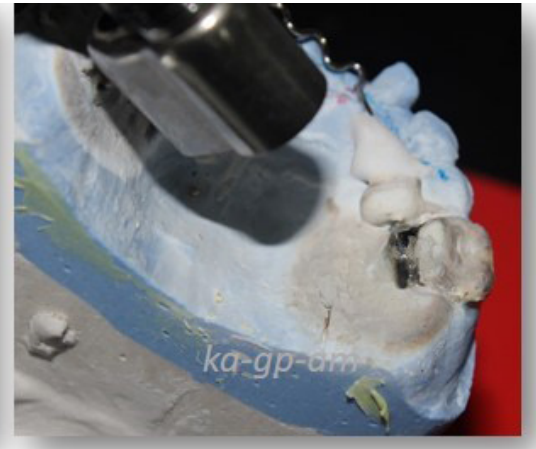

Figura 19. Debe existir un buen sellado de ambos elementos.

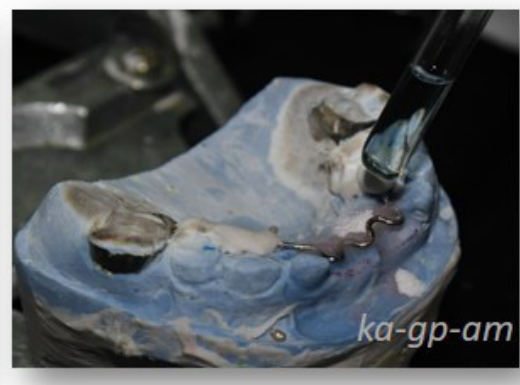

Figura 22. Colocar el líquido aplicando la técnica sal y pimienta.

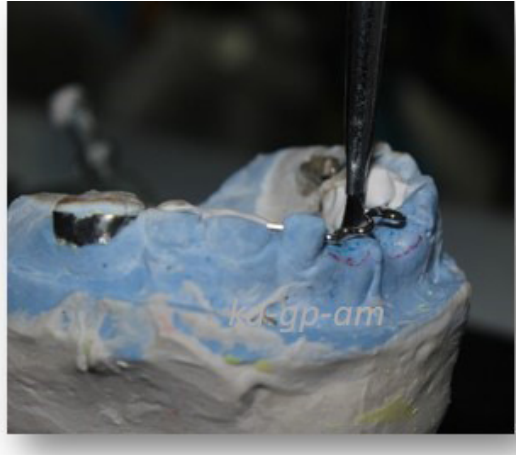

Figura 20. Colocar el aislante para acrílico en el sector anterior.

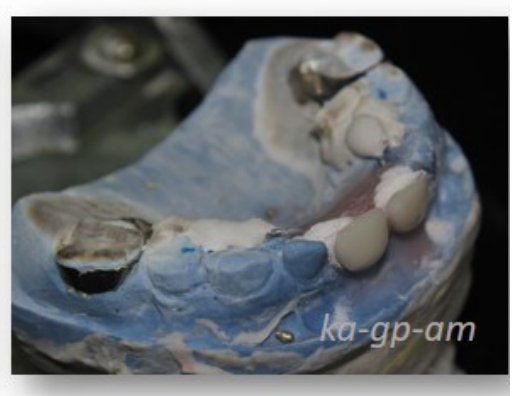

Figura 23. Colocar los dientes artificiales en correcta posición. 


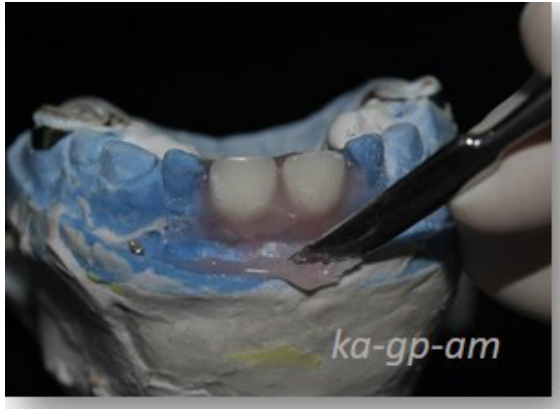

Figura 24. Retirar los excesos de acrílico de la zona vestibular.

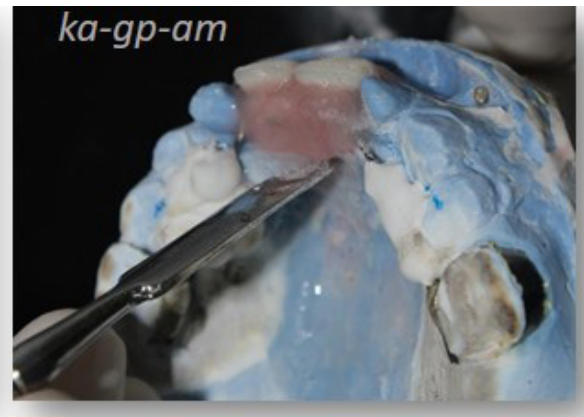

Figura 25. Retirar los excesos de acrílico de la zona palatina.

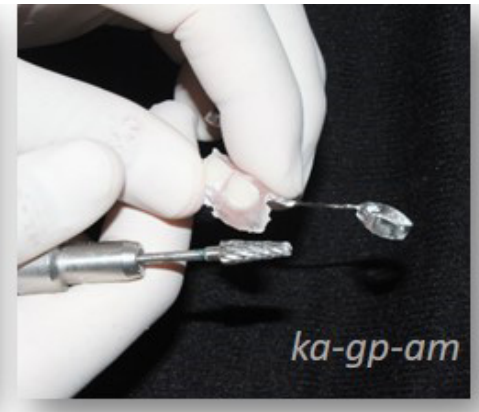

Figura 26. Recortar y pulir.

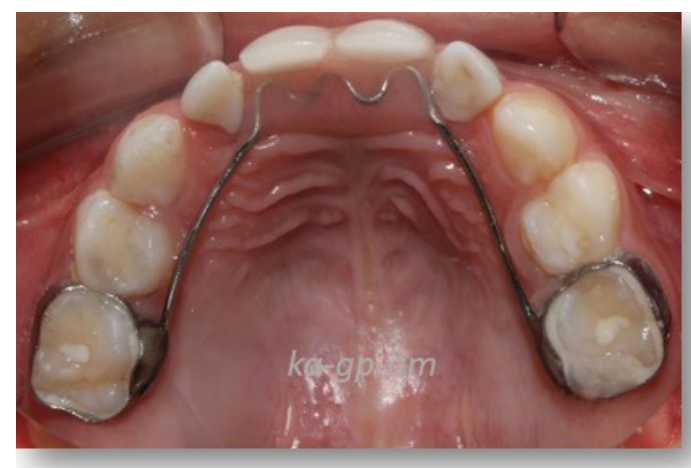

Figura 27. Cementación de prótesis con ionómero de cementación.

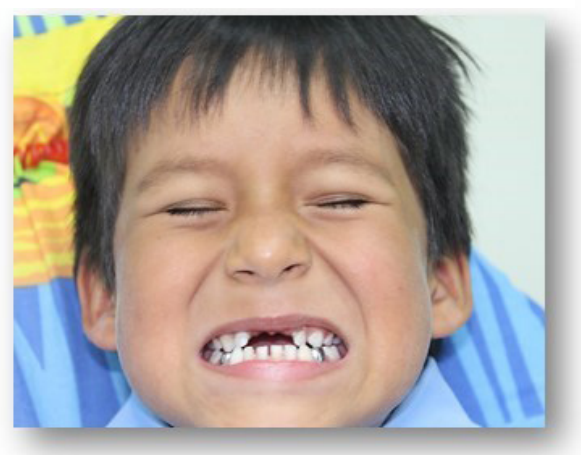

Figura 29. Vista frontal del paciente al momento del examen odontológico.

\section{DISCUSIÓN}

La rehabilitación bucal temprana es absolutamente necesaria para garantizar el desarrollo óseo y el crecimiento cráneofacial(21). Para el diseño de la prótesis se debe valorar cuidadosa los aspectos relacionados con el crecimiento y desarrollo de las arcadas dentarias. Desde los 3 años y 6 meses hasta los 5 años y 6 meses aproximadamente las dimensiones sagitales

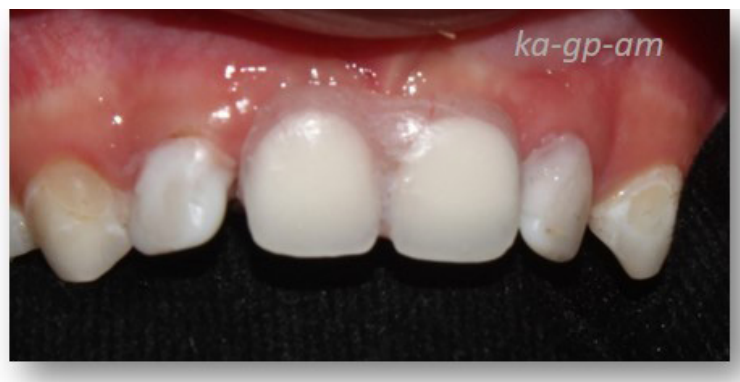

Figura 28. Sector Anterior con la prótesis ya instalada.

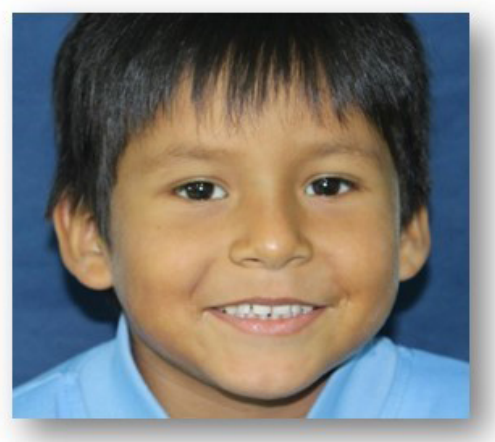

Figura 30. Vista frontal del paciente al momento de finalizar el tratamiento.

no registran un cambio significativo. Pero dentro de los 6 años hasta los 7 años y 6 meses, la dimensión intercanina de ambas arcadas aumentan $2 \mathrm{~mm}$ aproximadamente y la distancia intermolar, $1 \mathrm{~mm}$ en el maxilar y $0,5 \mathrm{~mm}$ en la zona mandibular(2).

La armonía facial es un factor primordial en el ambiente social en el que vive y se mueve el individuo, además de conducirle a tener un estado de ánimo 
satisfactorio y una valoración de su personalidad. Una sonrisa con unos dientes sanos resulta ser muy agradable y el poder abrir la boca con una seguridad de dominio es un factor positivo en las relaciones humanas. En el caso de los niños el presentar ausencia de dientes genera inseguridad, comportamientos violentos a causa de la baja autoestima(22-24).
La necesidad de rehabilitar o mejorar las funciones deterioradas, requiere de un control constante. Por lo tanto se debe revisar periódicamente, para determinar el cumplimiento de las indicaciones proporcionadas al paciente y poder realizar las correcciones que puedan requerir las prótesis. Se debe crear un compromiso entre padre, niño y odontopediatra para el éxito del tratamiento $(1,25,26)$.

\section{CONCLUSIONES}

1. La decisión a tomar para el uso de las prótesis debe estar basada ante todo en la experiencia clínica, el buen juicio, la disponibilidad de materiales protésicos adecuados, el conocimiento de las fases de crecimiento, desarrollo y los principios de oclusión. Niños con pérdida precoz están más motivados psicológicamente y funcionalmente a aceptar el tratamiento sin inconvenientes.

\section{AGRADECIMIENTOS}

CD. Ana Ximena Rivera Chávez y Mg. Esp. Úrsula María Dolores Albites Achata por su gentil ayuda en la toma de fotografías y su edición.

\section{REFERENCIAS BIBLIOGRÁFICAS}

1. Saavedra G, León C. Prótesis Pediátrica. En: Boj j, Catalá M, García - Ballesta C, Mendoza A, Planells P, editores. Odontopeditría La Evolución del Niño al Adulto Jovén. 1ra ed. España: Ripano;2012. p. 507-20.

2. Silva E. Prótesis en la Dentición Temporal. Perú, Imprenta de la FAP. 201.

3. Barbería E, Boj J, Catalá M, García C, Mendoza A. Odontopediatría. 2da ed. España: Masson;2002.

4. Villena R, Pachas F, Sánchez Y, Carrasco M. Prevalencia de caries de infancia temprana en niños menores de 6 años de edad, residentes en poblados urbano marginales de Lima Norte. Rev Estomatol Herediana. 2011; 21(2):79-86.

5. Villena R. Caries de infancia temprana. II Encuentro Peruano de Odontología para Bebes, Lima-Perú. Libro de resúmenes del congreso, 2006. [citado Febrero 2010]. Disponible en: http:/ /www.odontobebeperu.org/ acuerdos/02-01.pdf.

6. Begzati A, Berisha M, Meqa K. Early childhood caries in preschool children of Kosovo - a serious public health problem. BMC Public Health. 2010; 10:788.

7. Parisotto TM, Silva CMS, Oliveria CS, Santos MN, Gavião MBD. Prosthetic rehabilitation in a four-year-old child with severe early childhood caries: a case report. J Contemp Dent Pract. 2009; 10(2):1-8.

8. Zaror C, Martínez-Zapata MJ, Abarca J, Díaz J, Pardo Y, Pont À, Ferrer M. Impact of traumatic dental injuries on quality of life in preschoolers and schoolchildren: A systematic review and meta-analysis. Community Dent Oral Epidemiol. 2017;1-14.

9. Holan G, Needleman H. Premature lossof primary anterior teeth due to trauma: potencial short - and long - term sequelae. Dent 
Traumatol 2014; 30:100-6.

10.Vergo T. Prosthodontics for pediatric patients with congenital/ developmental orofacial anomalies: a long - term follow- up. J Prosthet Dent. 2001;86:342-7.

11. Morales r, Guevara J. Alteraciones Estructurales de los dientes. Kiru. 2010;7(2):83-90.

12. Abadie V, Couly G. Congenital feeding and swallowing disorders. En: Masdeu J, González G. Handbook of clinical neurology. Cambrigde, Estados Unidos; 2013. p. 1539-49.

13. Bessadet M, Nicolas E, Sochat M, Hennequin M, Veyrune JL. Impact of removable partial denture prosthesis on chewing efficiency. J Appl Oral Sci. 2013; 21(5):392-6.

14. Pereira L, Miasato JM. Mantenedor de Espaço Estético-funcional em Odontopediatria. Rev Odontol Univ Cid São Paulo. 2010; 22(2):154-62.

15. Derbanne M, Sitbon M, Landru M, et al. Case report: Early prosthetic treatment in children with ectodermal dysplasia. Eur Arch Paediatr Dent. 2010; 11:(6)301-5.

16. Silva E. ¿Cuándo y por qué el empleo en niños de la Prótesis Fija y Semi Fija en el Maxilar Superior? Odontol Pediatr. 2008; 7:(2)22-6.

17. Benavente J, Ghezzi L. Asociación entre el número de piezas dentarias del sector anterior-superior perdidas en forma prematura y los trastornos en la articulación de fonemas fricativos. Kiru 2007;4(1):8-13.

18. Quiroz O. Manual de Ortopedia Funcional de los maxilares y Ortodoncia Interceptiva. 2da ed. Colombia:Amolca;2000.

19. Guedes-Pinto A. Recursos protésicos en Odontopediatria. En Ferreira S, Biancala H, Guedes - Pinto A. Rehabilitación Bucal en Odontopediatría. Atención Integral. Colombia: Amolca; 2003. p. 229-268.

20. Santos da Silva V, Barcelos R, Ribero de Souza IP. Preceptions of pediatric patients and guardians about prosthetic appliances. J Clin Pediatr Dent. 2000;27:87-90.

21. Koch G, Poulsen S. Odontopediatria: abordaje clínico. 2a ed. Caracas: AMOLCA; 2011.

22. Cameron A. Manual de Odontopediatria. 3a ed. Rio de Janeiro: Elsevier; 2012.

23. Grando L, Da Fontoura L, Bernardi L, Pedroso G, Medeiros A, Borba F. Tooth supported prothetic rehabilitation in a 5-yearold child whit early childhood caries. J. Clin. Pediatr. Dent. 2007; 31(3): 173-6.

24. Corona A, Guerrero M, Rodríguez J, Pérez R, Hernández M. Rehabilitación oral en niños, con enfoque preventivo y psicológico: reporte de un caso. Rev Tamé. 2014; 3 (7):223-229.

25. Barrios Z, Salas M. Tratamientos protésicos en dentición primaria. Rev Odont de los Andes. 2006; 1 (2): 61-9.

26.Sheeba S, Deepak S. Functional and esthetic rehabilitation during deciduos dentition stage. Dent Res. J. 2011; 8(2): 108- 11

Recibido: 16 de abril de 2017

Aceptado: 12 de setiembre de 2017

Correspondencia: sharlot2989@hotmail.com 